\title{
Overcoming Pandemic Fatigue: How to Reenergize \& Motivate Ourselves \& Dental Teams at The Post-Covid Era
}

\author{
Ayse Basak Cinar* \\ Honorary consultant coach and researcher, University of Dundee, Dental School, Dundee, Scotland, UK
}

*Corresponding author: Ayse Basak Cinar, Honorary consultant coach and researcher, University of Dundee, Dental School, Dundee, Scotland, UK.

Received Date: February 08, 2021

Published Date: July 21, 2021

\section{Opinion}

Prolonged period of Covid-19 crisis is a physically and emotionally difficult time for dental team members. Not only are they feeling the strain of increased expectations, learning to work with the added discomfort, headaches, fatigue, and the increased body temperature additional PPE creates, but they also have real health fears of contracting the virus. Some of them may be questioning their future and/or how to work in near future with changing regulations.

Compounding this are the economic impacts of the crisis and more uncertainty about the future than many have ever experienced. Daily practices throughout the day, once easily achieved, may now lead to feel insurmountable when tensions and stress levels are high. Many team members experience fatigue due to all these challenges, regardless of being self-aware of it or not. Fatigue is when the tiredness is often overwhelming and is not relieved by sleep and rest [1]. NICE defines fatigue as Fatigue may be defined as severe mental and physical exhaustion, which differs from somnolence or lack of motivation, and which is not attributable to exertion or diagnosable disease [2]. Depression, lifestyle restrictions, increased number of leave/sick days from work, social isolation are few examples for the complications of fatigue [1.2]. Most importantly fatigue may bring team members one step closer to their breaking point [3].

Covid fatigue has been taking an enormous toll on healthcare workers, as addressed at the BMJ [4], and dental healthcare professionals are not exclusive of that reality. Actually, high levels of fatigue among healthcare professionals have been a concern over the years, as discussed by Paul Greig and Rosamund Snow [5]. The Covid-19 crisis has led to surge in mental wellbeing problems such as anxiety and stress, as highlighted by the British Dental Association Chair, Mick Armstrong [6]. The latest report by Dental Defence Union shows that $68 \%$ of UK dental professionals surveyed felt that their stress and anxiety levels had increased since the pandemic [7]. Several studies show that healthcare professionals working with patients during an epidemic/pandemic are at heightened risk of mental health problems in the short and longer term, particularly: psychological distress, insomnia, alcohol/ drug misuse, and symptoms of posttraumatic stress disorder, depression, anxiety, burnout, anger, and higher perceived stress [8]. Therefore, strategies and interventions targeting to improve the mental wellbeing of healthcare professionals should be proactive, future oriented, and prevention oriented.

Predictors of workplace mental wellbeing is multi-layered referring to social, organisational, individual, psychological, and environmental [8]. The magnitude and impact of every predictor vary from person to person, so workplace mental wellbeing interventions and policies should be agile and person-centred. As the British Dental Association Chair, Mick Armstrong mentions there is need for a system focused on prevention-based care, lowering stress for clinicians, and ensuring the best patient care [6]. Active engagement of both managers [6] and employees are essential in the workplace intervention development and implementation process [9]. CIPD highlights the need for placing 
employee wellbeing at the centre of business models and viewing it as the vital source of value creation, the dividends for organisational health can be significant [10].

5-staged Resilience and Agility model (Figure 1) stems from the successful outcomes of our international and local projects. Both projects have shown that the model's person-centered and proactive approach improves self-management skills for wellbeing (self-efficacy, self-esteem, resilience) and decreases the stress levels of the participants [11-13]. The model is originally a self-leadership development framework that integrates self-management and selfreflection dynamics to social engagement and collective purpose setting. Studies have shown that self-leadership is the ground for effective stress management and social communication, and successful performance [14]. We propose that the Model can serve as a guideline to design and execute wellbeing interventions for dental teams and organizations.

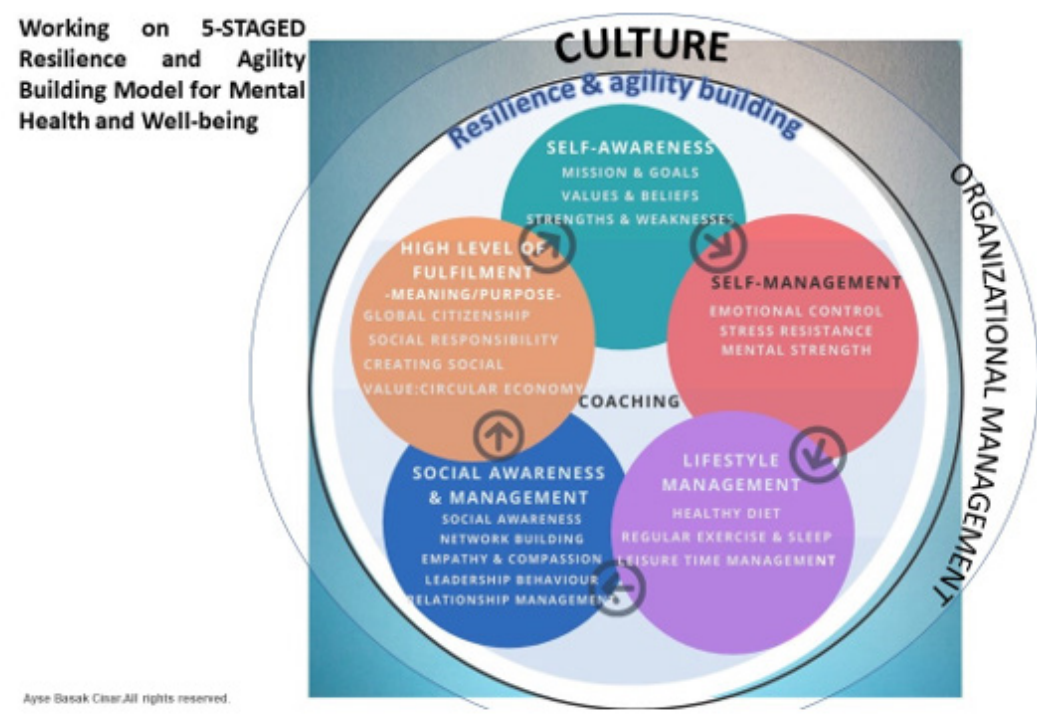

Figure 1

The key feature of the model is to improve systematically personal wellbeing and team wellbeing through 'One for All' approach. Tailored methodology in every stage of the Model enhances individual wellbeing, while on the other hand, it strengthens agile and resilient growth of teams. Thereby, it provides a methodological to build a workplace wellbeing culture. Wellbeing is not just for employees; it is for company leaders and CEOs as well. Creating a culture of wellbeing starts at the top and strengthened by the active participation of the employees. Studies show that employees who feel cared for and valued by their leaders and company have higher performance and less absenteeism at work. A Deloitte study identifies five pillars a workplace must constitute to deem itself a "Simply Irresistible Organisation"- meaningful work, supportive management, positive work environment, growth opportunity and trust in leadership [15]. It is also highlighted that employees look for employee wellbeing programmes focusing on employee, their families, and their entire experience at work and life. All that may highlight the need to design and execution of wellbeing interventions through collaborative participation of the managers and the employees. Those interventions also need to highlight the personal and professional growth of each individual, thus refers to the self-leadership journey of each employee and manager.
Such interventions are more important than ever for dental health care teams, in particular, if we think about their communication with and service provision for patients. The quality of care is directly related with the wellbeing, including mental health, of the whole team. At the post COVID-era many patients will have high levels of anxiety and stress about dental visits, and every member of the dental team will serve as a leading figure for secure, safe, and compassionate communication and dental healthcare. Therefore, understanding the dynamics of self-leadership and improving wellbeing, inclusive of mental wellbeing, seem to be one of the cornerstones of the sustainable future of dental health care services.

At the post-Covid-era, there is an emerging need for dental teams to feel reenergized and motivated and to emerge stronger, together. Positive and enhanced wellbeing, in particular mental wellbeing, is the key driver for that state for greater achievement and fulfilment. Dental team culture where everyone's self-leadership practice for wellbeing is supported and valued will be the key for successful dental organizations of the future.

\section{Acknowledgement}

None. 


\section{Conflict of Interest}

The authors declare no conflict of interest.

\section{References}

1. NHS What's the difference between tiredness and fatigue?

2. NICE, Tiredness/fatigue in adults.

3. Merete Wedell Wedellsborg (2020) How to Lead When Your Team Is Exhausted-and You Are, Too. Harvard Business Review.

4. Gerada C, Walker C (2020) Covid fatigue is taking an enormous toll on healthcare workers.

5. Greig P, Snow R (2017) Fatigue and risk: are train drivers safer than doctors? BMJ 359: j5107

6. Mick Armstrong (2020) The dental profession's mental health crisis: what needs to happen. BDA Blog.

7. Hannah Pugh (2020) Mental health and wellbeing in dentistry. Genera Dental Council Blog.

8. Stuijfzand S, Deforges C, Sandoz V, Sajin C, Jaques C, et al. (2020) Psychological impact of an epidemic/pandemic on the mental health of healthcare professionals: a rapid review. BMC Public Health:20.
9. Gray P, Senabe S, Naicker N, Kgalamono S, Yassi A, et al. (2019) Workplace-Based Organizational Interventions Promoting Mental Health and Happiness among Healthcare Workers: A Realist Review. Int J Environ Res Public Health 16(22): 4396.

10. CIPD [2020], Wellbeing at work.

11. Cinar AB, Oktay I, Schou L (2014) "Smile healthy to your diabetes": health coaching-based intervention for oral health and diabetes management. Clin Oral Investig 18(7): 1793-801.

12. Cinar AB, Freeman R, Schou L (2018) A new complementary approach for oral health and diabetes management: health coaching. Int Dent J 68(1): 54-64.

13. Cinar AB [2016] Person-centered Health Coaching in a Scottish Prison Population: Findings at Training Completion. Journal of Person-Centered Medicine: 6: 98-107.

14. Dolbier CL, Soderstrom M, Steinhardt MAS (2001) The relationships between self-leadership and enhanced psychological, health, and work outcomes. J Psychol 135(5): 469-85.

15. Deloitte [2019] Is your organization simply irresistible? 